\title{
E-Society - A Financial and Event Management System
}

\author{
Indra Priyadharshini ${ }^{\mathrm{a}, 1}$, Jasmine Gilda $\mathrm{A}^{\mathrm{b}}$, Sherin Glory $\mathrm{J}^{\mathrm{c}}$ and Mukhil $\mathrm{V}^{\mathrm{d}}$

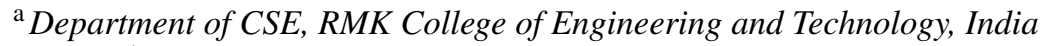 \\ ${ }^{\mathrm{b}}$ Department of CSE, R.M.K. Engineering College, India \\ ${ }^{\mathrm{c}}$ Department of CSE, Rajalakshmi Engineering College, India \\ ${ }^{\mathrm{d}}$ Department of CSE, RMK College of Engineering and Technology, India
}

\begin{abstract}
E-society - a financial and event management system, a web based application which leverage waterfall development model for managing the financial operations typically done in a house society and also provides the facility to create, organize and prioritize events and raise funds for the same. At present these details were maintained in a spreadsheet, and it has its own issues when comes to calculations, human introduced data error, missing required precession etc. Due to the manual maintenance of financial records, getting a spending report is become too tedious and difficult to extract in a given amount of time.This system is exclusively used by a small group or an organization allows people to keep track of the transaction between members of the society, admin and the workers working for that organization or society. By using this we can reduce the manual calculations and human errors while computation of expenditure. The system allows the retrieving and updating facilities to authorized persons. To bring in the transparency in expenses of the society's funds, the application allows every user to generate a report to know about the expenses and funds collected between a given date range.
\end{abstract}

Keywords. Create events, House Society, Transactions, Expenditure, Tracking, Transparency.

\section{Introduction}

The fountainhead of e-payment is, of policy, clicked to the nascence of the internet, which revolutionized the people like shrimp before. After all, if there were no people fat mesh, there would n't live online hoards and e-services. The chronicle of the internet starts in 1969 with ARPANET, the martial net which lived aimed to live a dispatch net in the Vietnam War epoch . But the main turning juncture passed in 1989 when Tim Berners - Lee mounted the answer of framing data royal to print and penetrate on the internet by harnessing the thereupon - sounded off " positions " or " go-betweens". In the nascence, the main instrumentalists on the e-payment demand were Millicent ( pioneered in 1995), ECash or CyberCoin (both in 1996) . The adultness of the original online indulgences were harnessing micro remitment complexes and their quotidian point lived the stab to enforce the electronic cash preferences ( parallel as e-money, digital cash or remem-

\footnotetext{
${ }^{1}$ Indra Priyadharshini, Department of CSE, RMK College of Engineering and Technology, India.

E-mail: indra.priyadharshini@gmail.com
} 
brances) [1]. besides, in 1994, Amazon is pioneered (one of the e-commerce frontiersmen) and Pizza Hut starts having online viands ladder . Can you swallow it? The original online quittance complex lived one measure earlier of all Pizza Hut 's contestants. Along with internet growth, frontiersman online remitment indulgences jumped to run in the original moiety of the $90 \mathrm{~s}$. They're website or app - grounded, which means there's no absence to baptize a different software or pick up alone outfit, which breathed the case a limited ages ago . presently complexes are accessible from any dodge catenated to the internet. Every age there are makeshift answers in the e-payments folks that stimulate e-commerce neoplasm . New instrumentalists manufacture electronic remittances both snap to apply and handy for fiends who recompense online.

\section{Related works}

In this neighborhood we will instead present a terse scrutiny of previouswork clicked to pecuniary and occurrence superintendence complex . savvy has strutted that the legend to manufacture the tracing and taking the finance snap is to solace the druggie with pictographic representation . thus the strategy of druggie interface .

The authors of [2-4] suggested that, to shirk revenue and outgo numbers and in the identical mores to remind a person, we evolve an android usage which may facilitative in all the statuses and it can live invested in our android phones. It prop us to remind and subjoin some data that what are the revenue comes from dissimilar persons and what are all the charges or compensations we've to compensate in distinct appointment or month. Due to the economy one could fin the slope in the education system and reforms [5-7]. Chandhini et al. bounced that, Income and Expense Tracker will conserve data of day-today, semiweekly, triweekly, semimonthly charges, Manages your charges and earnings in a naked and intuitive drag. Addict can choose family of charge, access dissimilar data like addict can come by photograph, adjoin locality, choose measure of charge etc. And this will deliver to the indigenous database. Addict can con and chum charge as per weekly, triweekly, semimonthly. By employing this, we can break the handmade calculi for their charges and celebrate the imprint of the charge. In this, addict can feed his gain to cipher his full charges per daylight and these consequences will kept for individualized addict. It'll breathe downhill for them to partake the bill in this shadow. This shadow complex provides an absorbed bloc of features to abet you to address your charges and cash flood. Emilie Combaz advanced that, Since the 1980s, several biggish foreign adjunct arms, akin as the World Bank, command advanced fused dollars-and-cents administration data complexes (IFMIS) as a belly component in habilitating open dollars-and-cents administration (PFM) in equatorial - earnings fatherlands (LICs). The anticipation is that IFMIS will fabricate data on open finances compleat, effective, assured and clear . nonetheless, these spendy complexes hourly give out to ascertain the swore boons . thus what serve we grasp about what has unriddled, what has gave out, and why? What assignments does the literature pinpoint? A splitting retrospect of scholarly and slaty literature uncovered a scarcity of stern science. The validation underpinning obtainable has a numeric of sins, and rulings and recommendations are opposite 


\section{Existing System}

In this segment we review conventional financial management applications and focus on the issues faced by the users of these applications and also their methodology as a reference. We closely examined the traditional ways of handling the payments without the help of online services and wrote down the findings. Financial management system could be found everywhere around us but these systems were build for large circle of people and can be used by everyone. Events cannot be organized with these systems for a specific purpose. Funds cannot be raised for a common cause by small group of members. These systems were common for everyone around the globe. If we would like to balance a income and expense for every month we've to try to to it manually but we can't do that for every and each month those that have tons of income and expenses, so to scale back the strain for the person and make easy to calculate the income and expense, this application has been such a lot helpful for an individual to avoid the manual way calculating his income and expenses. Existing system doesn't use the smart concepts which are used now each day . In existing, we'd like to take care of the Excel sheets, CSV etc. files for the user daily and monthly expenses. In existing, there's no intrinsically complete solution to stay a track of its daily expenditure easily. to try to to so an individual on keep a log during a diary or during a computer, also all the calculations must be done by the user which can sometimes leads to errors resulting in losses.

\subsection{Disadvantages of existing system}

- The existing system isn't user friendly because data isn't maintained efficiently.

- The existing projects won't have any reminder to stay an individual during a specific date, in order that is that the only drawback during which the rest isn't present.

- The exiting project are going to be an unpopulated data because it's some disadvantages by not alerting an individual for every and each month.

- But it are often wont to perform calculation on income and expenses to beat this problem we propose the new project.

\section{Proposed System}

People of this era virtually got comfy and absolutely glad with the thought of constructing things on-line. winding up method manually by standard strategies with sizeable quantity of human errors makes folks annoyed and area unit trying to find method to be automatic. conjointly they're within the look for facility of accessing the services from anyplace round the globe. therefore net based mostly solutions area unit extremely in demand. during this project we tend to propose an online application called "E-Society a monetary and event management system" that is useful to manage society's funds and expense as a daily or sporadically alternatively whenever we would like. It conjointly acts as AN indicator or hunter example within the quickest world that we tend to can't ready to keep in mind what the items we tend to had done.In this fast-moving world this net application are going to be terribly helpful for a folks—nation—land—country-people UN agency was a family and particularly for a business people. pursuit involves recording and analyzing the incomes and expenses of an individual or a corporation over a spe- 
cific amount of your time. Today, since we tend to reside in a very hurry up and obtain it done society, many folks area unit wanting forward to economical ways that to budget one's time and cash. throughout the recent years, some analysis has been administered on house budget. it's been noted that in most cases, event management is being done mentally and ne'er being placed on paper that makes expense pursuit terribly troublesome. because of some conflict or another stress we tend to forget some times that what area unit the financial gain or wherever the money needs to come back from or what the payments we've to pay.

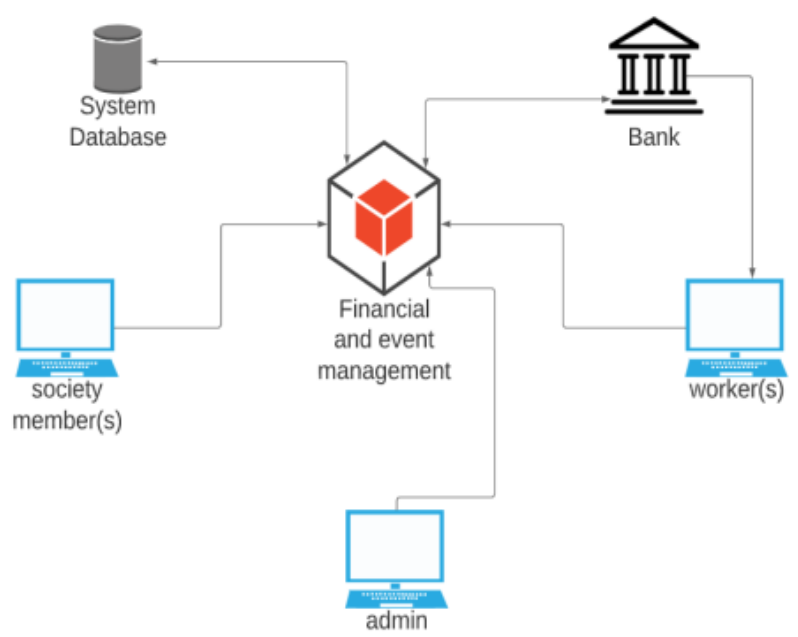

Figure 1. Architectural diagram of the proposed system

This new on-line financial gain and Expense hunter in JAVA can eliminate all the demerits that area unit found underneath the prevailing system. to cut back manual calculations, we tend to propose AN net based mostly application.Each user are going to be needed to register on the system at throughout registration, the user are going to be unambiguously known by their mail ID, which can be wont to maintain the record of every distinctive user. The project can keep a track of funding of a use and expense created by the association on on a daily basis to day basis. This application can generate reports simply inside few clicks [8]. the applying system will generate report between the offer date vary. it'll allow you to add the funding quantity to the repository(money vault). If we tend to exceed the target of our budget it's mechanically generate the notification that may sent via E-mail. AN email are going to be sent to the user at the tip of every month giving a quick outline of the monthly expenditure. The increaed security will help in user data safeguard $[9,10]$. because the application may be hosted on net the user has the privilege to access the applying from anyplace round the globe. the applying is developed mistreatment JAVA, MySQL, HTLM, CSS, Bootstrap and a few quantity of jQuery [11]. 


\subsection{Modules}

\subsubsection{Sign up Module}

Used to create an account for each user. This includes members, worker and admin. Details are stored in a table in database. New use has to enter their username, mail id, password and income if worker.

\subsubsection{Login Module}

Authorize and authenticate the user before letting them into the application. It is done by matching mail id and corresponding password.

\subsubsection{Dashboard}

This module shows the ongoing events for which the funds are being raised. Also shows the available money in the vault. And allows raise fund by paying, if member and get salary, if worker.

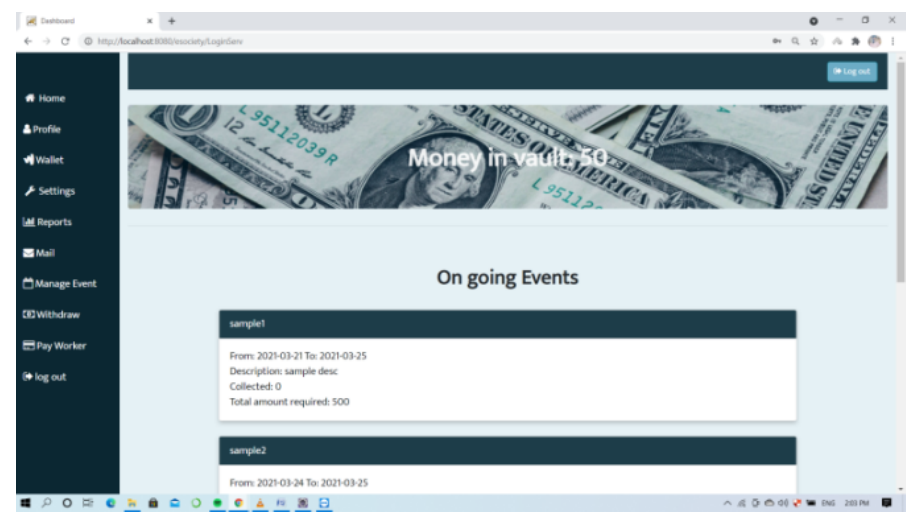

Figure 2. Dashboard

\subsubsection{User Profile}

This module allows the user to change name, password, income.

\subsubsection{Event creation}

New events can be created by entering event details like event name, date range for the event, event description and required funds for the event.

\subsubsection{Event modification}

Name, description, required funds and date range for the events can be changed.

\subsubsection{Report generation}

The investment and expense statements of the society can be viewed between the given date range. 


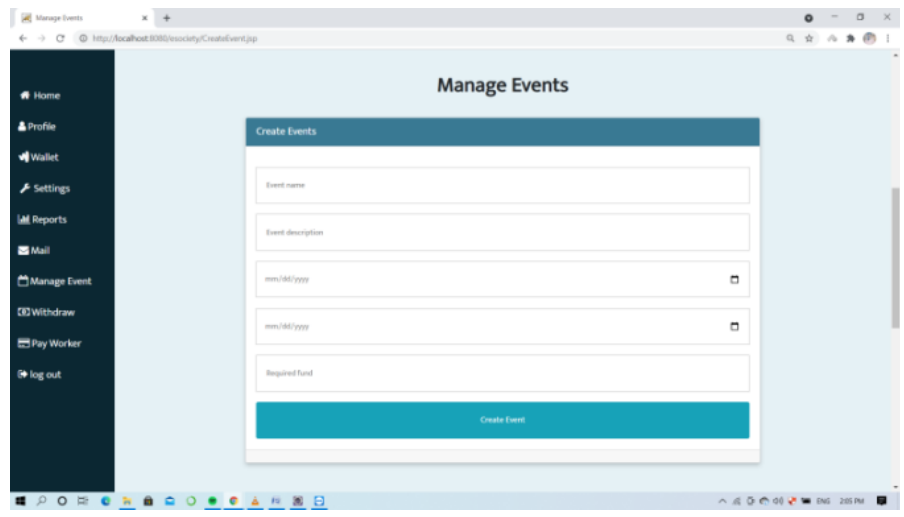

Figure 3. Event management section

\subsubsection{Withdraw funds}

Allows admin to withdraw funds.

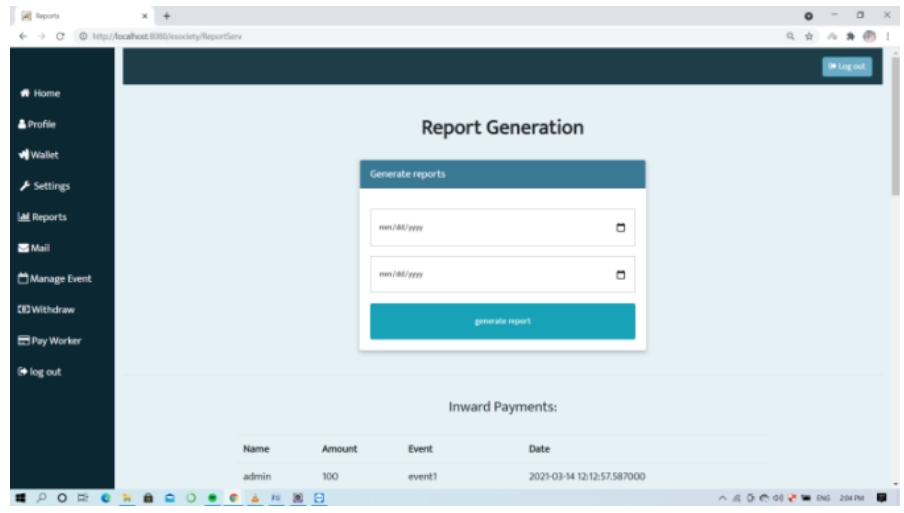

Figure 4. Report generation section

\subsection{Methodology used}

The methodology wont to develop this application is waterfall model. In "The Waterfall" approach, the entire process of software development is split into separate phases. during this Waterfall model, typically, the result of 1 phase acts because the input for subsequent phase sequentially. It is also mentioned as a linear-sequential life cycle model. it's very simple to know and use. during a waterfall model, each phase must be completed before subsequent phase can begin and there's no overlapping within the phases.

\subsection{Advantages of proposed System}

- The proposed system is user friendly as it has a simple to understand user interface.

- The users of the application are notified on monthly basis. 
- Easy management of events.

- Secure transmission of data.

- Exclusive for an organization.

- Easy tracking of money during event management

\section{Conclusion}

The proposed application using JAVA is with the obliged features addresses most of the problems faced by the manual financial management. This application has a good augmentation; it is especially strong from the point of view of relentless quality and flexibility. You can buy and incorporate large new features, modules, structures, or sections into the present application to make the general assignment a prevalent one from all points of view. The next version of this may be a mobile application with goodly improvement within the information security, user expertise, high computation powers and servers capable of handling requests at peak hours. Our resolution permits a user to search out out the matching users with the assistance of multiple servers while not revealing user profiles. Security analyses have shown that the new protocol achieves user profile privacy and user question privacy.

\section{Acknowledgement}

Support on demand, encouragement at the needed moment and guidance in the right direction are indispensable for the success of any project. We have received these in excess from all corners from various people, we are glad to submit our gratitude to them. We thank Shri. R.S. Munirathinam, Chairman, and Shri. R.M. Kishore, Vice Chairman of RMK group of Institutions for extending a generous hand in providing the best of resources to the college. Dr. T. Rengaraja, the esteemed Head of our Institution has been a source of motivation to all the staffs and students of our college. Our sincere thanks to Dr. D. PAULRAJ B.E., M.E., Ph.D., MISTE, the Head of the Department for his continuous support and motivation throughout our project. We extend our profound gratitude to Dr .M. VIGILSON PREM M.E., Ph.D, our Project Coordinator and Ms. INDRA PRIYADHARSHINI M.E., (Ph.D), our Guide for her guidance, who has indeed been a polestar throughout the course of the project, we thank her for giving us full support to complete the project successfully. Last, but not the least, we take this opportunity to thank all the staff members of the Department of Computer Science and Engineering. Regards to our family, classmates and friends who offered an unflinching moral support for completion of this project.

\section{References}

[1] SCN Education BV. Electronic Banking: The Ultimate Guide to Business and Technology of Online Banking. by SCN Education B.V, Springer-Verlag New York Incorporated,2001:402-9

[2] Bwoch G, Muwanga R. Financial Management and Accountability Reform. Uganda's Economic Reforms: Insider Accounts. 2009:338-54.

[3] Chêne M. The Implementation of Integrated Financial Management Systems (IFMIS). (2009) : 554-9. 
[4] Dorotinsky W, Watkins J. Government Financial Management Information Systems. InThe International Handbook of Public Financial Management 2013 (pp. 797-816). Palgrave Macmillan, London.

[5] Al Shobaki MJ, Abu-Naser SS. The Reality of Computerized MIS in the Palestinian Ministry of Education and Higher Education in Gaza Strip. International Journal of Engineering and Information Systems (IJEAIS). 2017 Aug;1(6):89-104.

[6] Abou-Dagga S, Elholy A. Quality enhancement of Palestinian higher education institutions: The case of Islamic University of Gaza (IUG). The Online Journal of Quality in Higher Education. 2014;1(2):53-9.

[7] Oberst BS, Jones RC. International trends in engineering accreditation and quality assurance. InThe Many Facets of International Education of Engineers 2020 Jul 26 (pp. 53-53). CRC Press.

[8] Goetze M, Strothotte T. An approach to help functionally illiterate people with graphical reading aids. InSmart Graphics Symposium UK 2001 (pp. 21-23). IBM TJ Watson Research Ctr.

[9] Harini N, Padmanabhan DT, Shyamala CK. Cryptography and security. Wiley India. 2011:786-91.

[10] Vaishnavi R, Anand J, Janarthanan R. Efficient security for Desktop Data Grid using cryptographic protocol. In2009 International Conference on Control, Automation, Communication and Energy Conservation 2009 Jun 4 (pp. 1-6). IEEE.

[11] Robbins JN. Learning Web Design Fourth Edition. O’Reilly Media. 2012: 116-26. 